\title{
The Effect of Segmental Stabilization Exercises on Pain, Disability and Static Postural Stability in Patients with Spondylolisthesis: A Double Blinded Pilot Randomized Controlled Trial
}

\author{
R. Choopani ${ }^{1}$, F. Ghaderi ${ }^{1}$, Z. Salahzadeh ${ }^{1}$, H. Adigozali ${ }^{1}$, H.N. Sivaki², E.M. Majd ${ }^{1}$, \\ M.R. Azghani ${ }^{3}$, A. Soltani ${ }^{1}$, M.A. Jafarabadi ${ }^{4,5}$ \\ 1 Department of Physiotherapy, Faculty of Rehabilitation, Tabriz University of Medical Sciences, Tabriz, Iran \\ 2 Department of Physiotherapy, School of Paramedical Sciences, Mashhad University of Medical Sciences, \\ Mashhad, Iran \\ 3 Biomechanics Department, Faculty of biomechanics Engineering, Sahand University of Technology, Tabriz, Iran \\ ${ }^{4}$ Road Traffic Injury Research Center, Tabriz University of Medical Sciences, Tabriz, Iran \\ ${ }^{5}$ Department of Statistics and Epidemiology, Faculty of Health, Tabriz University of Medical Sciences, Tabriz, Iran
}

CORRESPONDING AUTHOR:

Fariba Ghaderi

Tabriz University of Medical Sciences

Tabriz, Iran

E-mail: ghaderif@tbzmed.ac.ir

DOI:

10.32098/mltj.04.2019.18

LEVEL OF EVIDENCE: 3

\begin{abstract}
SUMMARY
Purpose. The aim of this study was to compare the effects of stabilization and general exercises on pain, disability and postural stability variables in patients with spondylolisthesis. Methods. 24 patients randomly assigned into the stabilization $(n=12)$ and general exercise $(n=12)$ groups. Both groups performed the exercises 2 times a week for a period of 2 months. Visual analogue scale (VAS) and Quebec back pain disability questionnaire were used to assess the pain and functional disability, respectively. Postural stability has been evaluated using a force platform with eyes opened and closed while standing on firm and foam base of support. The parameters were the mean total velocity $(\mathrm{M}-\mathrm{V})$ and the SD of velocity in sagittal $(\mathrm{SD}-\mathrm{Vy})$ and frontal $(\mathrm{SD}-\mathrm{Vx})$ plan.

Results. There was a significant decrease in VAS and Quebec in both stabilization and general exercise groups $(\mathrm{P}=0.001, \mathrm{P}<0.001$ for $\mathrm{VAS}$ and $\mathrm{P}=0.001, \mathrm{P}=0.001$ for Quebec respectively). From postural stability parameters only $\mathrm{SD}-\mathrm{Vx}$ has increases significantly in general exercise group $(\mathrm{P}=0.008)$. More increase was observed in $\mathrm{M}-\mathrm{V}$ variable in the stabilization group $(\mathrm{P}<0.001)$.

Conclusions. Pain and disability were improved in both stabilization and general exercise groups but postural stability variables was not changed in different directions in stabilization group.
\end{abstract}

KEY WORDS

general exercises; low back pain; postural stability; spondylolisthesis; stabilization exercises

\section{INTRODUCTION}

A condition in which a vertebra slips forward against the caudal one with or without the separation of posterior elements is called spondylolisthesis (SPL)(1). Approximately about two to six percent of the general population is affected by this disorder (2). SPL is a clinical expression of segmental instability, meaning that there is an increase in the range of the neutral zone around the spinal segment that may be caused by a trauma or degenerative changes (3).
Among the three subsystems providing spinal stability (including active, passive and neural), the active component (muscles) is very important when the others are disrupted. Different muscles can control and protect the spinal segments (4), which consist of the local and the global muscle stabilizer and mobilizers (5). Global muscles are generally targeted in conventional exercise therapy (6). However, the local stabilizers are thought to be more effective in the case of segmental instabilities such as SPL as 
they are very useful in preserving the suitable position of the lumbar segments during trunk movements (7). Studies investigating local stabilization exercises in SPL (7) or chronic LBP (8), have shown positive effects on pain and disability for these types of treatments.

The presence of chronic back pain also may impair the overall performance and neural system which in turn affects balance (postural stability) (9). Postural stability is described as the capability of controlling the center of mass (COM) regarding the base of support (BOS) (10). Pain or proprioceptive impairment associated with lumbar spine defects can cause stability deficiencies (11). Therefore, reduction in the pain and/or improvement of proprioception in these patients could have positive effects on regaining optimal postural balance.

The treatment approach for SPL includes non-surgical and surgical methods. Non-surgical treatment is the primary approach to SPL and may involve subcategories such as pain relief modalities, stabilization or general exercises, postural correction, and life style modification (12). It has been reported that exercise programs are the most effective component of non-surgical care in patients with SPL. Despite the positive outcomes of surgical treatment, patients undergoing surgery experience secondary complications, such as iatrogenic SPL, decreased vertebral arch and mobility, sagittal imbalance, and increased adjacent segment stress (transitional syndrome). Previous studies have suggested that non-surgical treatment may reduce the need for surgery in patients with SPL (12).

Some previous studies have shown postural stability deficits in LBP patients and the effect of stabilization exercises on postural stability in chronic LBP (13). Despite vast variety of studies investigating the exercises, choosing a specific type is still an issue for the clinicians and sometimes depends on their clinical opinions (14).

Therefore, the aim of this study was to compare the effects of segmental stabilization exercises along with general exercises on pain, disability and static postural stability in SPL patients.

\section{METHODS}

\section{Subjects}

A total of 120 LBP patients with the diagnosis of low grade SPL were referred by a neurosurgeon from 31 July 2016 till 7 Sep 2017 to the physiotherapy clinic of the rehabilitation faculty of Tabriz, Iran. The study protocol is approved by Ethics Committee of the Tabriz University of Medical Sciences, registered in IRCT (http://irct.ir/ ;
IRCT2016091029771N1). The study design was controlled by CONSORT checklist for RCTs and was conducted in accordance with the guidelines of the Declaration of Helsinki principles. Also, this study meets the ethical standard of the journal (15).

Patients were given verbal and written information about the study purpose and main steps. Those who signed the consent letter were included in the study provided that they met these criteria: (1) low back pain with or without pain in the lower extremities lasting more than three months (2) radiological diagnosis of grade one or two SPL and (3) aged between 20 to 60 years old male or female. Patients were excluded from the study if they had: (1) history of spinal surgery, (2) history of low back exercise therapy in the last three months, (3) pregnancy, (4) central nervous system (CNS) disorders (5) vestibular disorder (the participants were asked if they have dizziness and had been tested with repeated head rotation by the therapist). The flow diagram is shown in figure $\mathbf{1 .}$

\section{Interventions}

After enrollment, 24 subjects have met the inclusion criteria and were randomly assigned into two groups of stabilization exercises (SE) $(n=12)$ and general exercises (GE) $(n=12)$. Simple randomization method was made using blocks. Allocation to the groups was blind and random. Every patient was treated two sessions a week, lasting 8 weeks. The exercise protocol was done, supervised and progressed by a trained therapist. The patients followed the same protocol every day at home. Electrotherapy was done every session for both groups, including 20 - minute TENS (frequency $=100$ hertz, pulse duration $=70$ microseconds) and hot pack on the lower back. The study was conducted as a double blind method. The patients were blinded about the types of treatment and the assessor was a person who had not any role in training of the subjects.

\section{Stabilization exercises}

The objective of these exercises are to re-educate the stability muscles group (9). Pressure biofeedback was used to train each patient how to use their transversus abdominis in the exercises. It was necessary for the first session and then, the exercise protocol started which had three different stages with progression (table I). The patients had to hold each exercise about 5 to 10 seconds, three times a day and each time 10 repetitions for each exercise. Each session included an exercise to previous exercises for the patient to learn all the exercises within a month, then repeat them for the second month (9). 


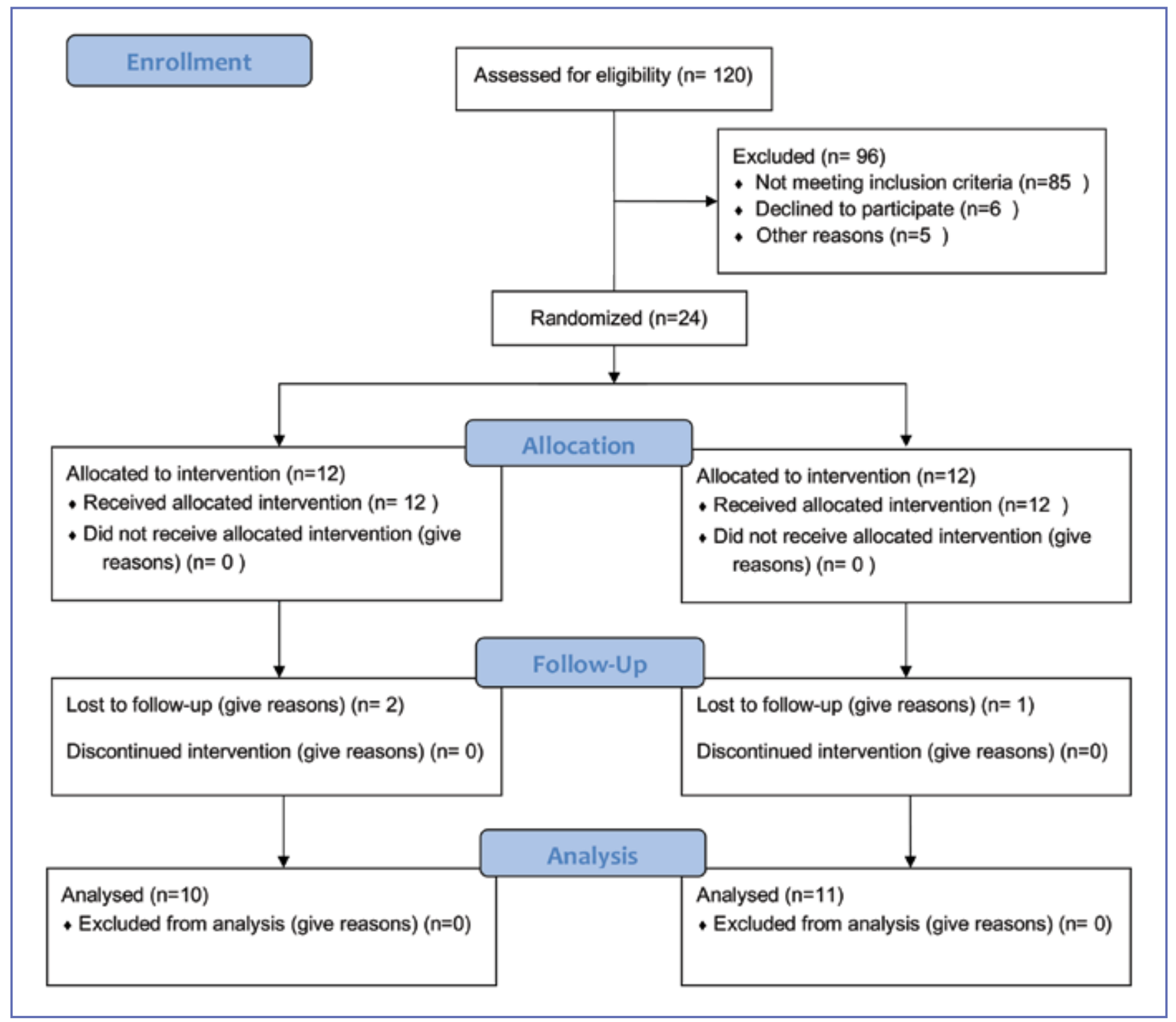

Figure 1. Flow diagram of the study.

Table I. Exercise protocol in SE group.

\begin{tabular}{ll} 
Segmental control in static position & $\begin{array}{l}\text { (1) isolated isometric multifidus contraction in prone } \\
\text { (2) sitting abdominal hollowing } \\
\text { (3) standing abdominal hollowing }\end{array}$ \\
\hline Segmental control in close chain movements & $\begin{array}{l}\text { (4) bridging with abdominal hollowing in crook lying } \\
\text { (5) } 30^{\circ} \text { squat with abdominal hollowing }\end{array}$ \\
\hline Segmental control in open chain movements & $\begin{array}{l}\text { (6) arm and leg movement with abdominal hollowing in supine } \\
\text { (7) arm and leg movement with abdominal hollowing in sitting } \\
\text { (8) leg movement with abdominal hollowing in standing }\end{array}$ \\
\hline
\end{tabular}




\section{General exercises}

Stretching, strengthening and flexion types of exercises were chosen for this group (16), since they are mostly known as general type (6). Stretching exercises were held 15 to 30 seconds, the others were held 5 to 10 seconds and each was done 10 times a day. The exercises included, (1) single and double knee to chest, (2) hip flexors stretch, (3) hamstring stretch, (4) piriformis stretch, (5) heel slide, (6) bridging, (7) partial and diagonal curl up and (8) leg cycling.

\section{OUTCOME MEASURES}

\section{Primary outcomes: (pain and disability)}

Pain and functional disability were assessed by Visual Analogue Scale (VAS) and Persian version of Quebec back pain questionnaire $(\mathrm{QBPQ})$, respectively before and after the intervention. The high reliability of these questionnaires have been reported (17).

\section{Secondary outcomes: Postural stability variables}

Static postural balance was measured using a force platform (Kistler, Version 4.0.x, and Type 2812 A, Switzerland). COP parameters in this study were mean total velocity $(\mathrm{M}-\mathrm{V})$, standard deviation of velocity in the anteroposterior direction $(\mathrm{SD}-\mathrm{Vy})$ and standard deviation of velocity in the mediolateral direction $(\mathrm{SD}-\mathrm{Vx})$. The procedure was explained to each patient and done in a quiet place with natural temperature. The patients had to stand barefoot, comfortable (not stiffening their body and avoiding unnecessary movements at the same time). The participants were asked to place foot with shoulder span at free angle and hands hanging beside the body. The subjects were asked to look only at the front. Five seconds were counted for the patients to adapt to the condition before starting recording the four conditions: Standing with eyes open (EO), eyes closed (EC) on firm and standing with $\mathrm{EO} / \mathrm{EC}$ on the foam base of support.

Before each test, the force plate had been calibrated and it was set at $200 \mathrm{~Hz}$ of sample frequency and $10 \mathrm{~Hz}$ low pass filter to prevent high frequency noise. Each recording lasted 30 seconds, three times for every condition and the patients were given one minute to rest between each condition.

\section{Statistical analysis}

Data analysis has been done using the SPSS software 16 (SPSS Inc., Chicago, Illinois, USA) at the 0.05 level of significance. The normality of the data was determined by Kolmogorov-Smirnov (K-S). Where the data was non-normal, logarithmic transformation was done before other analyses. To define the homogeneity of the two groups at the beginning of the study, independent t-test and chi-square tests, and for comparison of the parameters before and after the intervention in each group, the paired t-test were used. Linear mixed model as a covariance structure based on Autoregressive 1(AR1) was applied to analyze and compare COP variables. Estimation of the parameters was done by Restricted Maximal Likelihood Method (RMLM).

\section{RESULTS}

Twenty-four patients with grade one lumbar spondylolisthesis were enrolled, 21 of which completed the study. Descriptive statistics of patients is presented in table II. There were no significant differences between groups in demographic data at the beginning of the study. The data except for SD-Vx $(\mathrm{P}<0.05)$ had normal distribution $(\mathrm{P}>0.05)$.

\section{PRIMARY OUTCOMES (PAIN AND DISABILITY)}

There was a significant decrease for VAS in both the SE and GE after the intervention (mean dif. $=3.09 \pm 1.88, \mathrm{P}=$ 0.001 and mean dif. $=2.77 \pm 1.36, \mathrm{P}<0.001$ respectively), but between group analysis showed no significant difference (mean dif. $=0.6 \pm 0.394, \mathrm{P}=0.175)$ (table III).

The Quebec score showed a significant decrease after intra group analysis in the SE and GE (mean dif. $=18.2 \pm 12.39$, $\mathrm{P}=0.001$ and mean dif. $=19 \pm 12.6, \mathrm{P}=0.001$ respectively).

Table II. Patient's demographic information (N: 12 for each group).

\begin{tabular}{llll}
\hline Variable & $\begin{array}{l}\text { SE group } \\
\text { Mean }(\text { SD) }\end{array}$ & $\begin{array}{l}\text { GE group } \\
\text { Mean }(\text { SD) }\end{array}$ & P value \\
\hline Age & $48.1(14.51)$ & $51.36(6.75)$ & $0.51 ”$ \\
\hline Height & $161.2(0.07)$ & $158.13(0.06)$ & $0.312 "$ \\
\hline Weight & $74.94(7.59)$ & $77.37(11.62)$ & $0.581 "$ \\
\hline Sex & 8 female / 2 males & 10 female / 1 males & $0.586 \dagger$ \\
\hline "P value based on independent sample t-test. + P value based on Chi-square test. &
\end{tabular}


No difference was found between group for this variable (mean dif. $=1.8 \pm 4.93, \mathrm{P}=0.719)$ (table III).

\section{Secondary outcomes (Postural stability)}

In the SE group, SD-Vy variable, had an increase about $0.005 \mathrm{~m} / \mathrm{s}$ after intervention, which was not statistically significant $(\mathrm{P}=0.410)$. There was also a slight increase in other variables $\mathrm{M}-\mathrm{V}$ and $\mathrm{SD}-\mathrm{Vx}$, which was not significant $(\mathrm{P}=0.132$ and $\mathrm{P}=0.268$ respectively) (table IV).

In the GE group, there have been a significant increase about $0.109 \mathrm{~m} / \mathrm{s}$ in SD-Vx after intervention $(\mathrm{P}=0.008)$ (figure 2). A slight decrease was observed in $M-V$ and SD-Vy (in contrast with the SE group) which did not reach a significant level ( $\mathrm{P}=0.27$ and $\mathrm{P}=0.884$ respectively) (table $\mathrm{V}$ ).

Based on the Mixed Model Analysis after intervention, the mean of $\mathrm{M}-\mathrm{V}$ and $\mathrm{SD}-\mathrm{Vy}$ variables in SE group, were 0.017 and $0.011 \mathrm{~m} / \mathrm{s}$, respectively, more than that of GE group, which was statistically significant $(\mathrm{P}=0.012$ and $\mathrm{P}<0.001$ respectively) (figure 3 and 4 ).

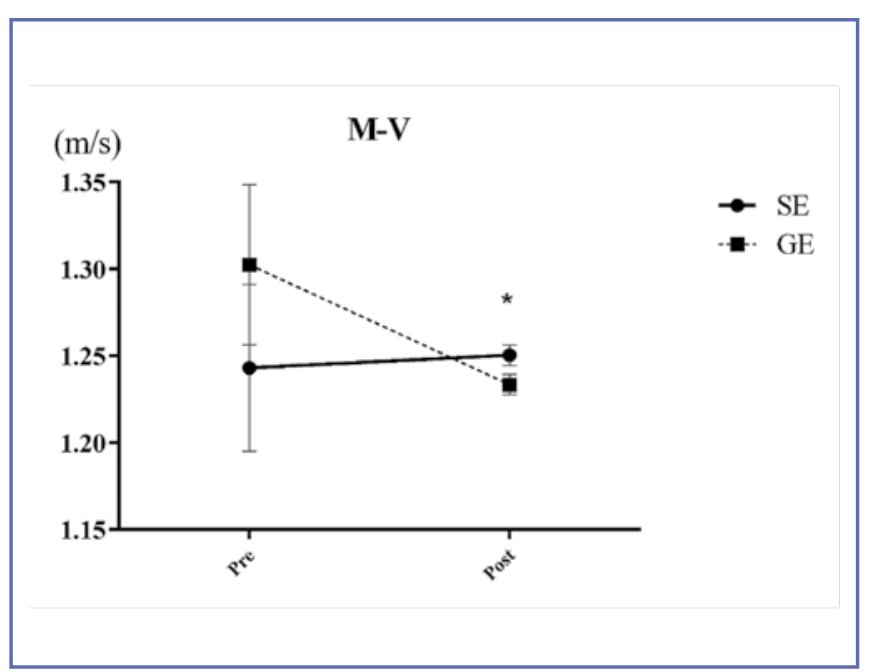

Figure 2. Comparison of mean total velocity (Mean and SE values) between groups SE and GE after the intervention based on Linear Mixed Model analysis. M-V, Mean total Velocity; SE, Stabilization Exercises; GE, General Exercises.

Table III. Pain and Disability at baseline and post intervention changes.

\begin{tabular}{llccc} 
& Variable & $\begin{array}{c}\text { Stabilization exercises } \\
(\mathbf{n = 1 0})\end{array}$ & $\begin{array}{c}\text { General exercises } \\
(\mathbf{n}=\mathbf{1 1})\end{array}$ & MD (95\% CI) between groups \\
\hline Pain & Before & $5.16(2.36)$ & $5.5(1.67)$ & $-.34(-2.2$ to 1.5$)$ \# \\
\hline After & $2.07(1.17)$ & $2.73(.86)$ & $-.559(-1.393$ to .276$)+$ \\
\hline Misability $(95 \%$ CI $)$ within groups $\dagger$ & $3.09(1.73$ to 4.44$) *$ & $2.77(1.85$ to 3.68$) *$ & $44.45(14.36)$ & $3.64(-15.02$ to 22.31$) \#$ \\
\hline & Before & $48.1(25.5)$ & $25.45(9.3)$ & $1.8(-8.66$ to 12.27$)+$ \\
\hline
\end{tabular}

CI, confidence interval;

$M D$, mean difference;

All values are presented as mean (SD)

$\dagger P$ value based on paired Student $t$ test.

\#P value based on independent Student t test

$¥ P$ value based on analysis of covariance adjusted for baseline measurements

*The mean difference is significant at the .05 level

Table IV. Adjusted Mean (Std.Error) for Pre-Post comparisons in SE group.

\begin{tabular}{lllll}
\hline Variables & & Mean (Std.Error) & Mean difference (95\% CI) & P value \\
\hline SD-Vy & Pre & $0.713(0.004)$ & $-0.004(-0.009$ to -0.000$)$ & 0.410 \\
\hline SD-Vx & Post & $0.718(0.004)$ & $0.026(-0.020$ to 0.072$)$ & 0.268 \\
\hline Pre & Post & $-1.299(0.079)$ & & $-0.006(-0.015$ to 0.001$)$ \\
\hline
\end{tabular}




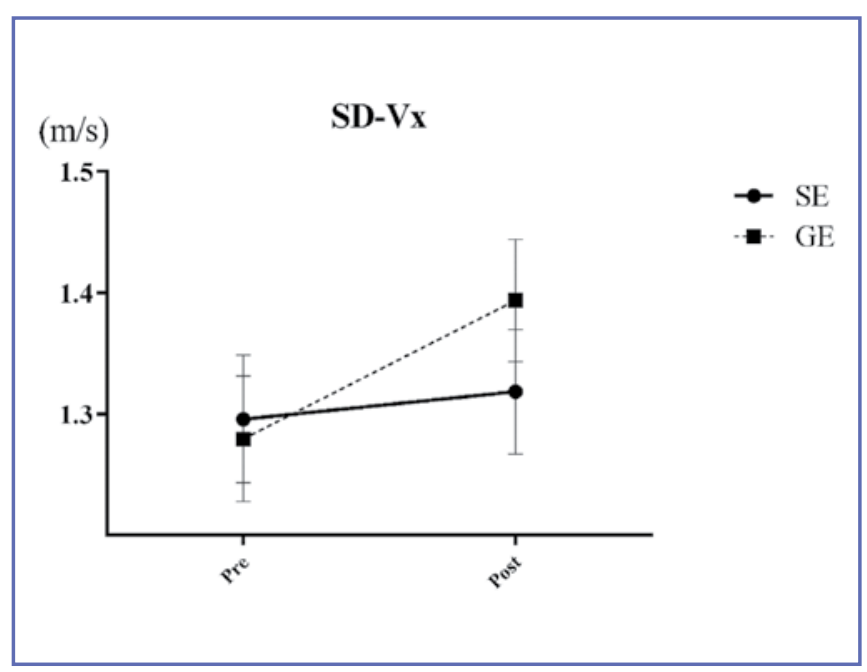

Figure 3. Comparison of SD of velocity in frontal plane (Mean and SE values) between groups SE and GE after the intervention based on Linear Mixed Model analysis (log transformation was done before the tests). SD-Vx, Standard deviation of Velocity in mediolateral direction; SE, Stabilization Exercises; GE, General Exercises.

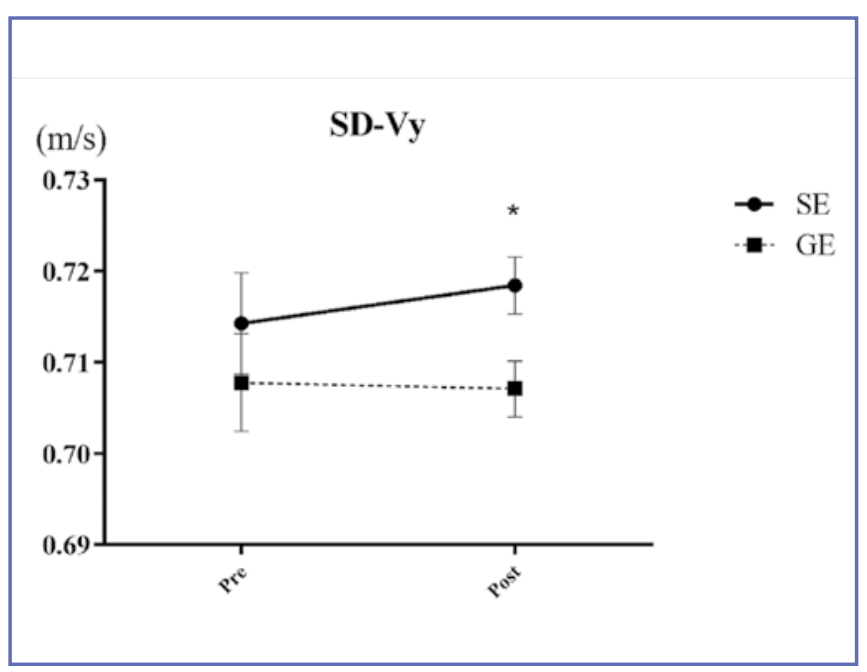

Figure 4. Comparison of SD of velocity in sagittal plane (Mean and SE values) between groups SE and GE after the intervention based on Linear Mixed Model analysis. SD-Vy, Standard deviation of Velocity in anteroposterior direction; SE, Stabilization Exercises; GE, General Exercises.

Table V. Adjusted Mean (Std.Error) for Pre-Post comparisons in GE group.

\begin{tabular}{lllll}
\hline Variables & & Mean $($ Std.Error) & Mean difference (95\% CI) & P value \\
\hline SD-Vy & Pre & $0.708(0.005)$ & $0.000(-0.009$ to 0.011$)$ & 0.884 \\
\hline SD-Vx & Post & $0.707(0.005)$ & & $0.109(0.029$ to 0.189$)$ \\
\hline $\mathrm{M}-\mathrm{V}$ & Pre & $-1.294(0.053)$ & & $0.008^{*}$ \\
\hline & Post & $-1.403(0.053)$ & $-0.407(-0.054$ to 0.193$)$ & 0.27 \\
\hline
\end{tabular}

P value based on Linear Mixed Model Analysis. This test is based on the linearly independent pairwise comparisons among the estimated marginal means. The mean difference is significant at the .05 level. SD-Vy, Standard deviation of Velocity in anteroposterior direction; M-V, Mean total Velocity; SD-Vx, Standard deviation of Velocity in mediolateral direction.

\section{DISCUSSION}

\section{Pain and disability}

The results of this study showed a significant decrease in pain and disability in both SE and GE groups after the intervention. There was more improvement in pain in the SE group compared to the GE group, and for disability in favor of GE group. However, it was not statistically significant, and therefore cannot be indicative of superiority of one type of exercise against another.

Clinically it seems that SE can be helpful in cases in which the segmental stability of the spine is damaged (7). According to Richardson and Jull (18), training the stability muscles with sub-maximal contraction can ameliorate pain and disability in patients with mechanical back pain. The protocol should be integrated and progressed to combine with main daily activities (18).

The results of the current study were similar to those of O'Sullivan et al. (1997) which concluded that stabilizing exercises improve pain and disability in SPL patients (7). In other similar studies there was a significant positive effect for pain $(19,20)$, and disability (19) for stabilizing exercises, as well as both stabilizing and conventional exercises in which the stabilization was superior (20). Contrary to their result, we observed no significant between-group differences after the intervention. Studies investigating 
chronic LBP have demonstrated a positive effect of the combination of SE and GE (13, 21, 22). Nevertheless, no difference has been observed between these treatment methods. The findings of the current study associated with pain and disability were in line with Cairns, Foster, and Wright (2006). The authors stated that, this lack of difference could give rise to increased stability in both groups, or increased general activity of participants (21).

As other studies, this study also had some limitations. The mean pain in both groups was moderate and a specific range of pain was not considered. Data from patients with low pain at the beginning of the study may have had an impact on the outcome. Follow up could be helpful in comparing the sustainability of the treatment effect between the two groups, which was not the case in this study. Also this was a pilot study, thus low sample size could limit the conclusion and may be a reason for the lack of a significant difference between groups. Since the majority of patients were female, the results of this study cannot be generalized to the whole society.

\section{Postural Stability}

Pain and disability questionnaires are subjective tools so they are not only related to the patient's physical recovery, but also a combination of emotions, culture, and patient beliefs (22). Therefore, it would be better if the effect of treatment is assessed by using other objective methods such as postural stability evaluation. In the present study, the velocity of the COP displacement significantly increased only in GE (SD-Vx) group but the trend of changes before and after intervention is different in two groups; mean velocity $(M-V)$ is decreased in GE group and increased in SE group (of course it was not statistically significant).

With the reduction of pain and disability, it was expected that the velocity of the COP displacement in SPL patients would be reduced, but the results contradicted this. One of the possible causes is the presence of rigidity in patients due to fear of pain-induced movement at the beginning of the study. According to Brumagne et al. (2008) COP may be controlled under simple test conditions in LBP patients, probably by developing a rigid posture or using more ankle strategy (23). As van Dieën et al. (2003) reported, increasing muscle activity in order to enhance the spinal stiffness, thereby improving its stability, is seen in LBP patients (24). Hence, after the treatment, pain relief might have made the patients stand somewhat more comfortable.

A recent study has reported the effects of back muscle weakness on degenerative spondylolisthesis and it showed weakness of back muscles can lead to or aggravate degenerative spondylolisthesis by increasing forward shear forces (25). So, back muscles strengthening exercise may improve spinal biomechanics from biomechanical point of view. Indeed, this study may help to prove this hypothesis from clinical point of view because we had strengthening component of back extensors in SE group and we had better results for reducing spinal stiffness and improving postural stability in SE group. On the other hand, increased muscular co-activation may reduce the ability and the rate of the body's response to perturbations of the environment, thus increasing the risk of falling (26). There should be a normal amount of exploratory movements for the COP to retain proper postural stability. Several studies have shown that there is reduced COP velocity in LBP patients compared to healthy subjects (27). So, it may not always be possible to state that the increase in the velocity of the COP means the diminution of the postural control and may also indicate the patient's ability to restore the COP to a more stable position faster. With that in mind, the SE group, have benefited a bit more from the treatment in case of postural stability.

Based on current knowledge, there are no other studies on postural stability in SPL patients. Previous studies in non-specific chronic low back pain patients, mostly have considered the increase in COP parameters as a reduction in postural stability (28). In view of that, these results were in contrast with another study which illustrate the positive effects of SE and/or GE on postural stability (29). After the intervention, an escalation was observed in all three velocity variables in the SE group. In the GE group, the increment was only in the frontal plane (which was significant), and there was a slight decrease in other two SD-Vy and $\mathrm{M}-\mathrm{V}$ variables. From this point of view, our results also contradicted the study of Koumantakis et al. (2005) who have indicated a significant improvement in the COP variables in favor of stability exercises, although they did not use the velocity parameters (22). There is more reliance on ankle than lumbar proprioception inputs (23), and improvement in a group of LBP in which the patients used more lumbar muscles inputs has been observed (30). Accordingly, it is inferred that an impairment in the proprioception via deficit in information from the lumbar multifidus (as one of the important sources of lumbar proprioception), the diminution of sensory information to the CNS (COP sway control will be less accurate) or alteration in muscle activation pattern, may cause a decrease in the ability of the CNS to change its strategy. Hence, when retraining these muscles, CNS may better adapt and switch the strategy, and better control of the COP velocity movements be provided.

Providing a detailed explanation and exercise training in both groups are the positive aspects of the present study 
which are not well achieved in the control group in some studies. In both stabilizing and general exercises, power, strength, and endurance must be considered (16). Stabilization exercises are integrated with individual's active daily living (ADL) in addition to these three items. It can reduce the stress to the spine arising from ADL before returning to work. In other words, it makes the spine ready to cope with these types of stress (16).

Muscle stretching, which has been performed in the GE group, can make the patients have an effective spinal alignment during other main exercises, thereby improving muscular and functional performance (16). These reasons may have contributed to the similarity between the results of the SE and GE in the current study. Some studies have reported that the SE increase the global and local muscles activity, and it is very difficult to isolate them (31). Koumantakis et al. (2005) stated that, accurate and precise performances of the SE are not feasible for all patients, while GE do not require much attention (22). In the current study, we checked the accuracy of the exercises in the clinic with a pressure biofeedback, but the patients had not the device at home, and it was not certain that how long and how correctly it has been done. This maybe another reason which has weakened other positive effects of SE.

\section{CONCLUSIONS}

In this study, there was no difference between groups after intervention; both SE and GE reduced pain and disability in low grade SPL patients and no change in postural stability parameters. To surely address the postural control changes, further studies with the Electromyography synchronizing can determine whether the increase or decrease of COP variables are in line with the improvement or worsening of symptoms.

\section{ACKNOWLEDGEMENTS}

We thank the patients who participated in this study for their co-operation. This article was written based on data for an MS thesis on physical therapy, which was registered at the Tabriz University of Medical Sciences, Iran.

\section{FUNDING}

This study was financially supported by Tabriz University of Medical Sciences.

\section{CONFLICT OF INTERESTS}

None of the authors declare a conflict of interest. 


\section{APPENDIX 1}

\section{TRAINING PROTOCOL}

The protocol lasted eight weeks. Patients visited the physiotherapy clinic two times a week. The first session began with exercise number one for patients in each group and every session one new exercise was added to the previous exercises. Five to ten seconds based on the patient's tolerance was counted for each exercise and the repetition was 10 times a day. All the exercises were finished in the first month and then, the 8 exercises were repeated for the second month.

Electrotherapy included a 20-minute TENS (frequency $=100$ Hertz, Pulse duration $=70$ microseconds) with a hot pack put on the lower back region. Correct postural mechanisms during daily activities were also taught for both groups.

\section{APPENDIX 2}

\section{STABILIZATION EXERCISE (SE) GROUP}

There are eight main exercises for this group which are classified in three subgroups. Patients in SE group learned two other basic exercises before the main protocol. This learning session included abdominal hollowing in the supine and prone position which was necessary for other main exercises.

\section{a) Abdominal hollowing in supine position}

Patient was in crook-lying position. Pressure biofeedback was put beneath the lower back (between L1 to S1) and set on the $40 \mathrm{mmHg}$. The Patient were taught to pull in his/ her stomach toward his/her back and head. No unneces- sary movement (e.g. pelvic tilt) should be seen and breathing must be gentle and normal during this exercise. Training was repeated till the patient learned the exercise (an increase of $10 \mathrm{mmHg}$ in pressure biofeedback). A slight contraction about 30 percent of maximal voluntary contraction (MVC), was sufficient and patient was asked to count to ten a bit loudly.

\section{b) Abdominal hollowing in prone position}

The patient was in prone position with the hands alongside the body. Pressure biofeedback was put beneath the abdomen in a way the umbilicus met the middle part, and the lower edges touched the anterior superior iliac spine (ASIS) in both sides. The patient was asked to be completely relax before beginning, so we could detect the amount of pressure change in relation to breathing. Then, the subject was pulling in his/her lower abdomen. The exercise was considered correct if: (1) 4-10 mmHg decrement, (2) no global muscle contraction should be seen (we checked this with the palpation of abdominal wall), (3) there should be no unnecessary movement in the pelvic and/or the spine. Patients were asked to do this exercise at home with a pillow beneath the abdomen till the first session.

\section{GENERAL EXERCISE (GE) GROUP}

This protocol consisted of 8 stretching, strengthening and flexion exercises.

(1) single and double knee to chest, (2) hip flexors stretch, (3) hamstring stretch, (4) piriformis stretch, (5) heel slide, (6) bridging, (7) partial and diagonal curl up and (8) leg cycling.

Table I. The stabilization exercises.

\begin{tabular}{ll}
\hline Segmental control in static position & $\begin{array}{l}\text { (1) isolated isometric multifidus contraction in supine and prone } \\
\text { (2) sitting abdominal hollowing } \\
\text { (3) standing abdominal hollowing }\end{array}$ \\
\hline $\begin{array}{l}\text { Segmental control in close chain } \\
\text { movements }\end{array}$ & $\begin{array}{l}\text { (4) bridging with abdominal hollowing in crook lying } \\
\text { (5) } 30^{\circ} \text { squat with abdominal hollowing }\end{array}$ \\
\hline $\begin{array}{l}\text { Segmental control in open chain } \\
\text { movements }\end{array}$ & $\begin{array}{l}\text { (6) arm and leg movement with abdominal hollowing in supine } \\
\text { (7) arm and leg movement with abdominal hollowing in sitting } \\
\text { (8) leg movement with abdominal hollowing in standing }\end{array}$ \\
\hline
\end{tabular}


Table II. The exercise therapy protocol for the SE group.

\begin{tabular}{|c|c|c|}
\hline $\begin{array}{l}\text { Session } \\
\text { number }\end{array}$ & Exercises & Time and Frequency \\
\hline 1 & 1) isolated isometric multifidus contraction in supine and prone & Three times a day, each time 10 repetitions \\
\hline 2 & $\begin{array}{l}\text { 1) isolated isometric multifidus contraction in supine and prone, 2) sitting } \\
\text { abdominal hollowing }\end{array}$ & Three times a day, each time 10 repetitions \\
\hline 3 & $\begin{array}{l}\text { 1) isolated isometric multifidus contraction in supine and prone, 2) sitting } \\
\text { abdominal hollowing, 3) standing abdominal hollowing }\end{array}$ & Three times a day, each time 10 repetitions \\
\hline 4 & $\begin{array}{l}\text { 1) isolated isometric multifidus contraction in supine and prone, 2) sitting } \\
\text { abdominal hollowing, 3) standing abdominal hollowing, 4) bridging with } \\
\text { abdominal hollowing in crook lying }\end{array}$ & $\begin{array}{l}\text { Three times a day, each time } 10 \text { repetitions } \\
\text { for each exercise }\end{array}$ \\
\hline 5 & $\begin{array}{l}\text { 1) isolated isometric multifidus contraction in supine and prone, 2) sitting } \\
\text { abdominal hollowing, 3) standing abdominal hollowing, 4) bridging } \\
\text { with abdominal hollowing in crook lying, 5) } 30^{\circ} \text { squat with abdominal } \\
\text { hollowing }\end{array}$ & $\begin{array}{l}\text { Three times a day, each time } 10 \text { repetitions } \\
\text { for each exercise }\end{array}$ \\
\hline 6 & $\begin{array}{l}\text { 1) isolated isometric multifidus contraction in supine and prone, 2) sitting } \\
\text { abdominal hollowing, 3) standing abdominal hollowing, 4) bridging } \\
\text { with abdominal hollowing in crook lying, 5) } 30^{\circ} \text { squat with abdominal } \\
\text { hollowing, 6) arm and leg movement with abdominal hollowing in supine }\end{array}$ & $\begin{array}{l}\text { Three times a day, each time } 10 \text { repetitions } \\
\text { for each exercise }\end{array}$ \\
\hline 7 & $\begin{array}{l}\text { 1) isolated isometric multifidus contraction in supine and prone, 2) sitting } \\
\text { abdominal hollowing, 3) standing abdominal hollowing, 4) bridging } \\
\text { with abdominal hollowing in crook lying, 5) } 30^{\circ} \text { squat with abdominal } \\
\text { hollowing, 6) arm and leg movement with abdominal hollowing in supine, } \\
\text { 7) arm and leg movement with abdominal hollowing in sitting }\end{array}$ & $\begin{array}{l}\text { Three times a day, each time } 10 \text { repetitions } \\
\text { for each exercise }\end{array}$ \\
\hline 8 & $\begin{array}{l}\text { 1) isolated isometric multifidus contraction in supine and prone, 2) sitting } \\
\text { abdominal hollowing, 3) standing abdominal hollowing, 4) bridging } \\
\text { with abdominal hollowing in crook lying, 5) } 30^{\circ} \text { squat with abdominal } \\
\text { hollowing, 6) arm and leg movement with abdominal hollowing in supine, } \\
\text { 7) arm and leg movement with abdominal hollowing in sitting, 8) leg } \\
\text { movement with abdominal hollowing in standing }\end{array}$ & $\begin{array}{l}\text { Three times a day, each time } 10 \text { repetitions } \\
\text { for each exercise }\end{array}$ \\
\hline $9-16$ & $\begin{array}{l}\text { Exercises were done in the remaining sessions as follows: } \\
\text { 1) isolated isometric multifidus contraction in supine and prone, 2) sitting } \\
\text { abdominal hollowing, 3) standing abdominal hollowing, 4) bridging } \\
\text { with abdominal hollowing in crook lying, 5) } 30^{\circ} \text { squat with abdominal } \\
\text { hollowing, 6) arm and leg movement with abdominal hollowing in supine, } \\
\text { 7) arm and leg movement with abdominal hollowing in sitting, 8) leg } \\
\text { movement with abdominal hollowing in standing }\end{array}$ & $\begin{array}{l}\text { Three times a day, each time } 10 \text { repetitions } \\
\text { for each exercise }\end{array}$ \\
\hline
\end{tabular}




\begin{tabular}{lll}
\hline $\begin{array}{l}\text { Session } \\
\text { number }\end{array}$ & Exercises & Time and Frequency \\
\hline 1 & 1) single and double knee to chest & $\begin{array}{l}\text { Three times a day, each time 10 repetitions } \\
\text { for each exercise }\end{array}$ \\
\hline 2 & 1) single and double knee to chest, 2) hip flexors stretch & $\begin{array}{l}\text { Three times a day, each time 10 repetitions } \\
\text { for each exercise }\end{array}$ \\
\hline 3 & $\begin{array}{l}\text { 1) single and double knee to chest, 2) hip flexors stretch, 3) hamstring } \\
\text { stretch }\end{array}$ & $\begin{array}{l}\text { Three times a day, each time 10 repetitions } \\
\text { for each exercise }\end{array}$ \\
\hline 4 & $\begin{array}{l}\text { 1) single and double knee to chest, 2) hip flexors stretch, 3) hamstring } \\
\text { stretch, 4) piriformis stretch }\end{array}$ & $\begin{array}{l}\text { Three times a day, each time 10 repetitions } \\
\text { for each exercise }\end{array}$ \\
\hline 5 & $\begin{array}{l}\text { 1) single and double knee to chest, 2) hip flexors stretch, 3) hamstring } \\
\text { stretch, 4) piriformis stretch, 5) heel slide }\end{array}$ & $\begin{array}{l}\text { Three times a day, each time 10 repetitions } \\
\text { for each exercise }\end{array}$ \\
\hline 6 & $\begin{array}{l}\text { 1) single and double knee to chest, 2) hip flexors stretch, 3) hamstring } \\
\text { stretch, 4) piriformis stretch, 5) heel slide, 6) bridging }\end{array}$ & $\begin{array}{l}\text { Three times a day, each time 10 repetitions } \\
\text { for each exercise }\end{array}$ \\
\hline 7 & $\begin{array}{l}\text { 1) single and double knee to chest, 2) hip flexors stretch, 3) hamstring } \\
\text { stretch, 4) piriformis stretch, 5) heel slide, 6) bridging, 7) partial and } \\
\text { diagonal curl up }\end{array}$ & $\begin{array}{l}\text { Three times a day, each time 10 repetitions } \\
\text { for each exercise }\end{array}$ \\
\hline 8 & $\begin{array}{l}\text { 1) single and double knee to chest, 2) hip flexors stretch, 3) hamstring } \\
\text { stretch, 4) piriformis stretch, 5) heel slide, 6) bridging, 7) partial and } \\
\text { diagonal curl up, 8) leg cycling }\end{array}$ & $\begin{array}{l}\text { Three times a day, each time 10 repetitions } \\
\text { for each exercise }\end{array}$ \\
\hline $\begin{array}{l}\text { Exercises were done in the remaining sessions as follows: } \\
\text { 1) single and double knee to chest, 2) hip flexors stretch, 3) hamstring } \\
\text { stretch, 4) piriformis stretch, 5) heel slide, 6) bridging, 7) partial and } \\
\text { diagonal curl up, 8) leg cycling }\end{array}$ & $\begin{array}{l}\text { Three times a day, each time 10 repetitions } \\
\text { for each exercise }\end{array}$ \\
\hline $9-16$ & \\
\hline
\end{tabular}

\section{REFERENCES}

1. Kalichman L, Hunter DJ. Diagnosis and conservative management of degenerative lumbar spondylolisthesis. European Spine Journal. 2008;17(3):327-35.

2. Osterman K, Schlenzka D, Poussa M, Seitsalo S, Virta L. Isthmic spondylolisthesis in symptomatic and asymptomatic subjects, epidemiology, and natural history with special reference to disk abnormality and mode of treatment. Clin Orthop Relat Res. 1993(297):65-70.

3. Blanda J, Bethem D, Moats W, Lew M. Defects of pars interarticularis in athletes: a protocol for nonoperative treatment. Journal of spinal disorders. 1993;6(5):406-11.

4. Hides JA, Jull GA, Richardson CA. Long-term effects of specific stabilizing exercises for first-episode low back pain. Spine. 2001;26(11):e243-e8.

5. Ghorbanpour A, Azghani MR, Taghipour M, Salahzadeh Z, Ghaderi F, Oskouei AE. Effects of McGill stabilization exercises and conventional physiotherapy on pain, functional disability and active back range of motion in patients with chronic non-specific low back pain. Journal of physical therapy science. 2018;30(4):481-5.

6. Lehtola V, Luomajoki H, Leinonen V, Gibbons S, Airaksinen $\mathrm{O}$. Efficacy of movement control exercises versus general exercises on recurrent sub-acute nonspecific low back pain in a sub-group of patients with movement control dysfunction. Protocol of a randomized controlled trial. BMC musculoskeletal disorders. 2012;13(1):55.
7. O'Sullivan PB, Phyty GDM, Twomey LT, Allison GT. Evaluation of specific stabilizing exercise in the treatment of chronic low back pain with radiologic diagnosis of spondylolysis or spondylolisthesis. Spine. 1997;22(24):2959-67.

8. Cho H-y, Kim E-h, Kim J. Effects of the CORE exercise program on pain and active range of motion in patients with chronic low back pain. Journal of physical therapy science. 2014;26(8):1237-40.

9. O'Sullivan PB. Lumbar segmentalinstability': clinical presentation and specific stabilizing exercise management. Manual therapy. 2000;5(1):2-12.

10. Shumway-Cook A, Woollacott MH. Motor control: translating research into clinical practice: Lippincott Williams \& Wilkins; 2007.

11. Moseley GL, Nicholas M, Hodges PW. Pain differs from non-painful attention-demanding or stressful tasks in its effect on postural control patterns of trunk muscles. Experimental brain research. 2004;156(1):64-71.

12. Demir-Deviren S, Ozcan-Eksi EE, Sencan S, Cil H, Berven S. Comprehensive non-surgical treatment decreased the need for spine surgery in patients with spondylolisthesis: Three-year results. Journal of back and musculoskeletal rehabilitation. 2019(Preprint):1-6.

13. Muthukrishnan R, Shenoy SD, Jaspal SS, Nellikunja S, Fernandes S. The differential effects of core stabilization exercise regime and conventional physiotherapy regime on postural control parameters during perturbation in patients with move- 
ment and control impairment chronic low back pain. BMC Sports Science, Medicine and Rehabilitation. 2010;2(1):13.

14. McGill SM. Low back exercises: evidence for improving exercise regimens. Physical therapy. 1998;78(7):754-65.

15. Padulo J, Oliva F, Frizziero A, Maffulli N. Basic principles and recommendations in clinical and field science research: 2018 update. Muscle Ligaments Tendons J. 2018;8(3):305-7.

16. Kisner C, Colby LA, Borstad J. Therapeutic exercise: foundations and techniques: Fa Davis; 2017.

17. Mousavi SJ, Parnianpour M, Mehdian H, Montazeri A, Mobini B. The Oswestry disability index, the Roland-Morris disability questionnaire, and the Quebec back pain disability scale: translation and validation studies of the Iranian versions. Spine. 2006;31(14):E454-E9.

18. Ferreira PH, Ferreira ML, Maher CG, Herbert RD, Refshauge K. Specific stabilisation exercise for spinal and pelvic pain: a systematic review. Australian Journal of Physiotherapy. 2006;52(2):79-88.

19. Nava-Bringas TI, Hernández-López M, Ramírez-Mora I, Coronado-Zarco R, Israel Macías-Hernández S, Cruz-Medina E, et al. Effects of a stabilization exercise program in functionality and pain in patients with degenerative spondylolisthesis. Journal of back and musculoskeletal rehabilitation. 2014:27(1):41-6.

20. ALI MF, ELWARDANY SH, ABDALRAHEEM RM. Isolated Lumbar Stabilization Exercises Versus Dynamic Lumbar Strengthening Exercises in Patients with Spondylolisthesis. Med J Cairo Univ. 2014;82(2):115-22.

21. Cairns MC, Foster NE, Wright C. Randomized controlled trial of specific spinal stabilization exercises and conventional physiotherapy for recurrent low back pain. Spine. 2006;31(19):E670-E81.

22. Koumantakis GA, Watson PJ, Oldham JA. Trunk muscle stabilization training plus general exercise versus general exer- cise only: randomized controlled trial of patients with recurrent low back pain. Physical therapy. 2005;85(3):209-25.

23. Brumagne S, Janssens L, Janssens E, Goddyn L. Altered postural control in anticipation of postural instability in persons with recurrent low back pain. Gait \& posture. 2008;28(4):657-62.

24. van Dieën JH, Cholewicki J, Radebold A. Trunk muscle recruitment patterns in patients with low back pain enhance the stability of the lumbar spine. Spine. 2003;28(8):834-41.

25. Zhu R, Niu W-x, Zeng Z-l, Tong J-h, Zhen Z-w, Zhou S, et al. The effects of muscle weakness on degenerative spondylolisthesis: A finite element study. Clinical Biomechanics. 2017;41:34-8.

26. Kang HG, Lipsitz LA. Stiffness control of balance during quiet standing and dual task in older adults: the MOBILIZE Boston Study. Journal of neurophysiology. 2010;104(6):3510-7.

27. Salavati M, Mazaheri M, Negahban H, Ebrahimi I, Jafari AH, Kazemnejad A, et al. Effect of dual-tasking on postural control in subjects with nonspecific low back pain. Spine. 2009;34(13):1415-21.

28. Palmieri RM, Ingersoll CD, Stone MB, Krause BA. Center-ofpressure parameters used in the assessment of postural control. Journal of Sport Rehabilitation. 2002;11(1):51-66.

29. Hwangbo G, Lee C-W, Kim S-G, Kim H-S. The effects of trunk stability exercise and a combined exercise program on pain, flexibility, and static balance in chronic low back pain patients. Journal of physical therapy science. 2015;27(4):1153-5.

30. Claeys K, Brumagne S, Dankaerts W, Kiers H, Janssens L. Decreased variability in postural control strategies in young people with non-specific low back pain is associated with altered proprioceptive reweighting. European journal of applied physiology. 2011;111(1):115-23.

31. Stevens VK, Coorevits PL, Bouche KG, Mahieu NN, Vanderstraeten GG, Danneels LA. The influence of specific training on trunk muscle recruitment patterns in healthy subjects during stabilization exercises. Manual therapy. 2007;12(3):271-9. 